\title{
IMPACTO DE LA RELACIÓN DE AYUDA DE CUIDADORES PRIMARIOS EN LA CALIDAD DE VIDA DE PACIENTES CON CÁNCER AVANZADO
}

\author{
IMPACT OF THE RELATIONSHIP PRIMARY CAREGIVERS HELP IN THE QUALITY OF \\ LIFE OF PATIENTS WITH ADVANCED CANCER
}

\author{
Alejandra Caqueo-Urízar' , Pablo Segovia-Lagos' , Úrsula Urrutia-Urrutia' , Claudia Miranda \\ Castillo ${ }^{2}$ y Edgar Navarro Lechuga ${ }^{3}$ \\ ' Universidad de Tarapacá, Chile \\ 2 Universidad de Valparaíso, Chile. \\ ${ }^{3}$ Universidad del Norte, Colombia
}

\section{Resumen}

Introducción: Este estudio evaluó el impacto de la figura del cuidador primario en la calidad de vida (CV) de pacientes con cáncer avanzado.

Método: El diseño de investigación es exploratorio descriptivo-correlacional y el muestreo no probabilístico por conveniencia con un error de estimación no superior al $5 \%$. La muestra estuvo compuesta por 34 pacientes diagnosticados con cáncer avanzado y sus respectivos cuidadores primarios, todos ellos pertenecientes a una unidad hospitalaria especializada en tratamientos paliativos. Se administraron los instrumentos FACT-G, SF-36 y la escala de sobrecarga del cuidador de Zarit.

Resultados: Los resultados indican que la CV global de los pacientes oncológicos es percibida negativamente, lo que sugiere que los problemas de salud física y emocional deterioran el funcionamiento físico cotidiano y las actividades sociales del mismo. Por su parte, los cuidadores valoran positivamente su CV; no obstante aquellos que proveen de cuidados a pacientes en estadios de funcionalidad más avanzados de la enfermedad presentan un leve grado de sobrecarga en las áreas física, social, psíquica y económica de su vida. Asimismo, se concluyó que la CV del paciente, en la dimensión estado funcional, se ve afectada positivamente por las dimensiones función social,
Abstract

Introduction: This study evaluated the impact of the primary caregiver on the quality of life (QL) of patients with advanced cancer.

Method: The research design is exploratory descriptive-correlational with non-probabilistic sampling for convenience with error not exceeding $5 \%$. The sample was composed of 34 patients diagnosed with advanced cancer and their respective primary caregivers, all of whom from a hospital unit specialized in palliative treatment. The instruments FACT-G, SF-36 and Zarit's caregiver overload scale were used.

Results: The results show that the overall QL of cancer patients is perceived negatively, which suggests that physical and emotional health problems deteriorate the patient's daily physical and social activities. With regard to caregivers, these rate their own QL positively; however those who provide care to patients with a more advanced state of cancer present a slight amount of overload in the physical, social, psychological and economic aspects of their lives. As such, the study concluded that the patient's functional state dimension is positively affected by the caregivers's social functions, welfare and vitality.

Conclusions: it can be observed that indeed, the level of deterioration in the functional and physical dimensions of the patient has an

\section{Correspondencia:}

Pablo Segovia Lagos

Avenida Carmen 390 departamento 609 Santiago de Chile, Chile

E-mail: pablosegovia_@hotmail.com; pabloseg@ucm.es 
bienestar y vitalidad del cuidador.

Conclusiones: Se constata que, efectivamente, el nivel de deterioro en las dimensiones física y funcional del paciente influye en la percepción que éstos tienen de su CV, así como también que los cuidadores de pacientes en estadios más críticos de la enfermedad se ven sobrecargados, probablemente, debido al rol más activo que deben asumir.

Palabras Claves: Calidad de vida, cáncer, pacientes oncológicos, cuidadores primarios. influence on the perception that they have of their QL, in addition to caregivers of patients in the more critical stages of the illness regarding themselves as overloaded, probably due to the more active role that they have to take on.

Keywords: Quality of life, cancer, cancer patients, primary caregivers.

\section{INTRODUCCIÓN}

Las estadísticas mundiales indican que el cáncer es una de las principales causas de muerte en todo el mundo. En concordancia con esto, en el año 2008 esta enfermedad causó 7,6 millones de defunciones, equivalente al $13 \%$ del total registrado $^{(1)}$. De igual forma, se espera que en el año 2030 se alcance una prevalencia del $27,7 \%$ en Chile, considerándose, de esta forma, un problema de salud pública ${ }^{(2)}$, alcanzando la segunda causa de muerte durante los últimos 30 años y duplicando su importancia relativa en ese periodo ${ }^{(3)}$. Sin duda, una enfermedad que requiere para su diagnóstico y tratamiento de una infraestructura multidisciplinar muy compleja, donde el síntoma más prevalente y de mayor trascendencia para las personas y sus familias, es el dolor ${ }^{(3,4)}$. En concordancia, el control de éste es uno de los principales indicadores de calidad asistencial utilizados por la OMS, no obstante, estudios recientes revelan que un importante número de profesionales de atención primaria no utilizan la morfina, o bien, no lo hacen correctamente. Pese a esto, no hay que olvidar que el $90 \%$ de este tipo de pacientes experimentan dolor, siendo en muchos casos la principal razón del deterioro de la calidad de vida en sus últimos días y un motivo importante de sus visitas a urgencias e incluso de que acudan a morir al hospital(5).

El cáncer terminal es una enfermedad avanzada, gradual e irreversible que no responde a los tratamientos médicos curativos; a su vez, va acompañada de un malestar general que afecta la esfera biológica, psíquica y social del paciente, así como también a los miembros de su familia, originando así alteraciones en la calidad de vida. Junto a esto, con el envejecimiento se produce la declinación de múltiples variables fisiológicas, que se traducen, a su vez, en la reducción de la reserva funcional. En congruencia, disminuye la tolerancia al estrés producido por la enfermedad y por los tratamientos de Quimioterapia, Radioterapia y Cirugía( ${ }^{(6)}$.

El Eastern Cooperative Oncology ${ }^{(7)}$ clasifica, mediante la Escala de Performance Status, los estadios de funcionalidad en pacientes con cáncer terminal, lo cual es posible observar en la tabla 1.

Esta escala y sus criterios son utilizados por los médicos e investigadores para evaluar cómo está progresando, a nivel funcional, la enfermedad de cáncer en un paciente, evaluar la forma en que afecta las habilidades de la vida diaria y determinar el tratamiento adecuado y el pronóstico de la misma ${ }^{(7)}$. Allí radica la relevancia de dicho indicador, ya que permite hacer distinciones respecto a las áreas de la cali- 


\section{Tabla 1. Clasificación de los estadios de funcionalidad en pacientes con cáncer terminal, según la Escala Performance Status}

\begin{tabular}{|c|l|}
\hline Estadio & \multicolumn{1}{|c|}{ Características } \\
\hline 0 & $\begin{array}{l}\text { Paciente plenamente activo, en condiciones de realizar todas las actividades, } \\
\text { sin restricción. }\end{array}$ \\
\hline 1 & $\begin{array}{l}\text { Paciente restringido en actividades físicamente extenuantes, pero capaz de } \\
\text { realizar actividades del diario vivir, recibe atención médica ambulatoria. }\end{array}$ \\
\hline 2 & $\begin{array}{l}\text { Paciente que recibe atención ambulatoria menos del 50\% del tiempo, capaz } \\
\text { de proveerse autocuidado, pero incapaz de Ilevar a cabo cualquier tipo de } \\
\text { trabajo. }\end{array}$ \\
\hline 3 & $\begin{array}{l}\text { Paciente capaz de proveerse autocuidado sólo en forma limitada, confinado } \\
\text { a la cama o silla más del 50\% de las horas de vigilia, necesita cuidados de } \\
\text { enfermería. }\end{array}$ \\
\hline 4 & $\begin{array}{l}\text { Paciente completamente discapacitado, postrado, no puede proveerse auto- } \\
\text { cuidado y necesita cuidado de enfermería. }\end{array}$ \\
\hline
\end{tabular}

dad de vida de estos pacientes que se ven afectadas en mayor medida, tanto en los que se ubican en estadios iniciales, como también los que se hallan en estadios más avanzados. En congruencia con lo antes mencionado, se presume que éstos últimos presentan mayores dificultades en su desempeño diario, así como también un evidente deterioro de sus capacidades y habilidades.

Ser diagnosticado de cáncer en fase terminal afecta de múltiples formas a la persona que lo padece y también genera alteraciones en gran parte del núcleo familiar. En la mayoría de los casos, es una sola persona quien asume el cuidado del enfermo, la cual debe ocuparse de las necesidades y demandas que éste requiere. Dicha persona recibe el nombre de cuidador principal y, comúnmente, es un miembro de la familia quien asume este rol $^{(8)}$. Este cuidador se define como la persona (familiar o allegado) que posee una condición favorable para asumir esta responsabilidad, la cual además cuenta con buena disposición para atender al paciente, mantiene un vínculo afectivo con él o ella, cuenta con una adecuada com- prensión para transmitir la información e indicaciones del equipo médico, así como también la facultad de entregar seguridad y bienestar emocional al paciente. Sin embargo, no siempre el cuidador primario podrá contar con dichas cualidades para ejercer su labor de cuidador, ya que estas características se apegan a un ideal preestablecido, el cual no necesariamente se debe cumplir a cabalidad.

En las últimas décadas se ha trabajado fuertemente en el concepto de calidad de vida, un aspecto fuertemente importante, el cual ha sido entendido como la percepción del individuo, de su posición en la vida en el contexto de la cultura y sistemas de valores en los que vive y en relación con sus objetivos, expectativas, estándares y preocupaciones ${ }^{(9)}$. También la literatura señala la calidad de vida como la habilidad de participar plenamente en funciones y actividades relacionadas con aspectos físicos, sociales y psicosociales apropiadas para la edad(10). Ésta implica una evaluación subjetiva de la satisfacción y/o insatisfacción que posee una persona en relación a una multiplicidad de factores, los cuales, además, se interrelacionarían 
entre sí. Junto a esto, se debe considerar que calidad de vida es una evaluación de carácter temporal y está ligada al estado de salud actual del sujeto ${ }^{(11,12)}$.

El cuidador de un enfermo de cáncer terminal necesita adaptarse al estrés que genera la demanda del cuidado, de hecho el cuidador debe asumir el cuidado de su paciente las 24 horas del día ${ }^{(13)}$, lo que provoca altos niveles de desgaste físico y psicológico ${ }^{(14,15)}$. Dicha tarea puede incluso dar origen a lo que hoy en día se conoce como Síndrome del cuidador primario, el cual está relacionado directamente con la sobrecarga que, a nivel físico como emocional, pueden padecer quienes ejercen este desgastante papel ${ }^{(16,17)}$. Por otra parte, la relación entre estados emocionales (ansiedad y depresión) del cuidador, el deterioro funcional del enfermo y el tiempo que el cuidador lleva prestando cuidados, es altamente significativa ${ }^{(18)}$. Es por ello que los cuidadores precisan de apoyo significativo, ya que atender a un enfermo representa un cambio sustancial en sus propias vidas. Requieren de educación respecto a la enfermedad y cuidados de salud mental, puesto que esta labor conlleva desgaste emocional ${ }^{(19)}$.

El rol del cuidador principal puede generar efectos positivos en el paciente, ya que éste último se sentiría apoyado, acogido y querido por su cuidador, además de proveerle de ayuda en el proceso de su enfermedad $^{(20)}$. En relación a lo anterior, un estudio sobre la calidad de vida en pacientes con cáncer de colon ${ }^{(21)}$ encontró que el nivel de calidad de vida de los miembros de la familia y/o cuidadores explicaba de mejor manera el nivel de calidad de vida del paciente, que las variables sociodemográficas o médicas. En relación a lo anterior, se presume que un cuidador puede ejercer positivamente su rol si se le brinda ayuda, si acepta relevos para su descanso, tener tiempo para sí mismo, si procura seguir haciendo una vida normal y mantenga una relación activa con su círculo social(19).

En los últimos años, ha cobrado importancia el estudio del rol de los cuidadores primarios y cómo éste influye en la calidad de vida de pacientes con cáncer en fase terminal. Esto puesto que se ha constatado que una de las dimensiones que más influye en la calidad de vida en pacientes oncológicos es el apoyo social que reciben éstos ${ }^{(22,23)}$.

Investigaciones en torno al tema ${ }^{(24)}$ sugieren que es esencial ampliar las evaluaciones de calidad de vida a los cuidadores de enfermos de cáncer, ya que ellos son el apoyo primario de éstos, y porque su grado de ajuste a la situación de enfermedad parece tener un efecto significativo sobre el ajuste del paciente.

Asimismo, el apoyo emocional que puede entregar un cuidador al paciente con cáncer en fase terminal, sirve como un amortiguador para que este último pueda afrontar los eventos estresantes que implica padecer esta enfermedad. De igual forma, dicho apoyo emocional es aun más importante durante esta fase, ya que el paciente al perder su autonomía, requiere de los cuidados que le puede otorgar su cuidador ${ }^{(25,26)}$.

Se observa que los aspectos más relacionados con la reducción de calidad de vida en el paciente son, en primer lugar, los aspectos físicos $y$, en segundo lugar, los aspectos emocionales ${ }^{(25)}$. Frost, et al. ${ }^{(27)}$ señalan que los pacientes que se encuentran en etapas avanzadas de un cáncer de mama perciben negativamente su estado de salud, presentan mayores problemas físicos y emocionales, así como un mayor deterioro en su vida cotidiana.

Este estudio tiene como objetivo establecer el impacto de la relación de ayuda de los cuidadores primarios sobre la calidad de vida de pacientes con cáncer en fase terminal de una población de la ciudad de Arica. 


\section{MÉTODO}

\section{Diseño de la Investigación}

El diseño de la investigación es exploratorio descriptivo-correlacional.

\section{Sujetos}

La muestra está conformada por 34 pacientes diagnosticados con cáncer avanzado y sus respectivos cuidadores primarios. Ambas muestras son atendidas en la Unidad del Dolor y Cuidados Paliativos del Hospital Regional Dr. Juan Noé Crevani de la ciudad de Arica.
Para la selección de la muestra definitiva se consideraron los siguientes criterios de inclusión: pacientes diagnosticados con cáncer en fase terminal que se encontraran en los estadios 2, 3 y 4 de la escala de funcionalidad Performance Status, ausencia de alteraciones mentales, ausencia de trastornos en el lenguaje, conocimiento del diagnóstico $y$, finalmente, que el paciente contara con un cuidador a cargo de él. El muestreo es no probabilístico por conveniencia con un error de estimación no superior al 5\% ( $p<0,05)$.

Las características sociodemográficas y clínicas de la muestra de pacientes y cuidadores se señalan en la tabla 2 y 3 , respectivamente.

Tabla 2 Características sociodemográficas y clínicas de la muestra de pacientes con cáncer en fase terminal

\begin{tabular}{|l|c|c|}
\hline \multicolumn{1}{|c|}{$\begin{array}{c}\text { Características Sociodemográficas } \\
\text { Muestra pacientes }\end{array}$} & Frecuencia & Porcentaje \\
\hline Sexo & 10 & $29,4 \%$ \\
Masculino & 24 & $70,6 \%$ \\
\hline Femenino & & \\
\hline Edad & 5 & $14,7 \%$ \\
30-49 & 13 & $38,2 \%$ \\
50-69 & 16 & $47,1 \%$ \\
70-89 & & \\
\hline Estado civil & 3 & $8,8 \%$ \\
Soltero & 19 & $55,9 \%$ \\
Casado & 5 & $14,7 \%$ \\
Separado & 7 & $20,6 \%$ \\
Viudo & 2 & \\
\hline Nivel educacional & 16 & $5,9 \%$ \\
Ninguno & 12 & $47 \%$ \\
Básico & 4 & $35,3 \%$ \\
Medio & & $11,8 \%$ \\
Técnico & 20 & \\
\hline Estadio de Funcionalidad (PS) & 7 & $58,8 \%$ \\
PS 2 & 7 & $20,6 \%$ \\
PS 3 & & $20,6 \%$ \\
PS 4 & & \\
\hline
\end{tabular}


Tabla 3. Características sociodemográficas de la muestra de cuidadores y/o familiares

\begin{tabular}{|l|c|c|}
\hline \multicolumn{1}{|c|}{$\begin{array}{c}\text { Características Sociodemográficas } \\
\text { Muestra cuidadores y/o familiares }\end{array}$} & Frecuencia & Porcentaje \\
\hline Sexo & 4 & $11,8 \%$ \\
Masculino & 30 & $88,2 \%$ \\
Femenino & & \\
\hline Edad & 6 & $17,6 \%$ \\
$20-29$ & 15 & $44,1 \%$ \\
$30-49$ & 12 & $35,3 \%$ \\
$50-69$ & 1 & $2,9 \%$ \\
$70-89$ & & \\
\hline Estado civil & 10 & $29,4 \%$ \\
Soltero & 18 & $52,9 \%$ \\
Casado & 3 & $8,8 \%$ \\
Separado & 2 & $5,88 \%$ \\
Viudo & 1 & $2,9 \%$ \\
Conviviente & & \\
\hline Nivel educacional & 7 & 20,6 \\
Básico & 10 & $29,4 \%$ \\
Medio & 8 & $23,5 \%$ \\
Técnico & 9 & $26,5 \%$ \\
Superior & & \\
\hline
\end{tabular}

\section{Recolección de datos}

La investigación se desarrolla en la Unidad del Dolor y Cuidados Paliativos perteneciente al Hospital Dr. Juan Noé Crevani de la ciudad de Arica. Dicha Institución de salud es el único centro sanitario de carácter público de la ciudad que atiende población con cáncer en fase terminal, así como también el único que cuenta con las condiciones y equipamiento para satisfacer las necesidades de los pacientes con dichas características. Las prestaciones otorgadas, tanto a los pacientes, como a sus familiares $y / 0$ cuidadores, se implementan y canalizan a través de la Unidad del Dolor y Cuidados Paliativos, por tanto es la entidad especializada en el abordaje de la temática objeto del presente estudio.
La solicitud de autorización para llevar a cabo la investigación se realizó, en primera instancia, a la médico jefe de dicha unidad, la enfermera jefe y la profesional psicóloga, quienes otorgaron el consentimiento entregando algunas pautas éticas de ejecución, tales como el manejo de la confidencialidad, que el primer contacto con la muestra se llevará a cabo en la unidad y, por último, solicitaron una devolución de información, al término del estudio, acerca de los datos arrojados.

El procedimiento que se llevó a cabo para realizar dicha selección consistió en revisar las fichas clínicas de cada paciente y posterior a esto los investigadores asistieron a los controles clínicos para determinar el estado de salud contractual de cada paciente y si éste estaba apto para la apli- 
cación de los instrumentos. Asimismo, por medio de esta entrevista clínica, se realizó el contacto con los pacientes y/o cuidadores para solicitar su colaboración en la presente investigación. Una vez que éstos firmaron el consentimiento informado se procedió a la administración de la batería de instrumentos.

La metodología utilizada para administrar la batería de instrumentos a la muestra de esta investigación, fue la siguiente: (a) autoadministración de los instrumentos cuando los pacientes y sus cuidadores principales estaban en condiciones físicas (no estar postrado o débil físicamente) e intelectuales (saber leer y escribir) de hacerlo; (b) administración de los instrumentos por los investigadores cuando los pacientes y sus cuidadores principales no cumplían con los requisitos mínimos de autoaplicación (capacidad física e intelectual). El lugar en el cual se realizó la aplicación de los instrumentos fue en el domicilio de los pacientes.

Los instrumentos utilizados para la evaluación fueron los siguientes:

En pacientes se utilizaron las siguientes escalas:

1. Escala de Evaluación Funcional de la Terapia del Cáncer FACT-G ${ }^{(28)}$ creado por Cella, Tulsky, Gray, Sarafian, Linn, Bonomi et al., 1993: Evalúa la calidad de vida de pacientes con cualquier tipo de cáncer y sirve para conocer el impacto del tratamiento. Está conformada por 34 ítems con escalas tipo Likert, con un rango de 0 a 4. Estas subescalas son: Estado físico general de la salud, ambiente familiar y social, estado emocional y capacidad de funcionamiento personal.

2. Cuestionario de Salud SF-36 ${ }^{(29)}$ creado por Ware et al., 1992. Adaptada al castellano por Alonso et al., 1995: Permite evaluar la salud percibida de la población y su evolución en el tiempo, y sirve, asimismo, de referencia para la comparación de las diferencias en el estado de salud (calidad de vida) según los tratamientos y/o monitorizar procesos de tratamiento. Los ítems del cuestionario informan de estados tanto positivos como negativos de la salud física y del bienestar emocional. Tiene 8 subescalas: Función física, rol físico, dolor corporal, salud general, vitalidad, función social, rol emocional y salud mental. Estas subescalas se agrupan en tres áreas de salud general: (a) Estado Funcional (compuesta por las subescalas Función Física, Función Social, Rol Físico y Rol Emocional), (b) Bienestar (compuesta por las subescalas Vitalidad, Salud Mental y Dolor Corporal) y (c) Evaluación General de la Salud (compuesta por la subescala Salud General y el ítem $\mathrm{n}^{\circ} 2$ del instrumento).

En los cuidadores principales se utilizó:

3. Escala de Sobrecarga del Cuidador de Zarit( ${ }^{(30)}$, creada por Zarit, Reever y Bach-Peterson, 1980. Adaptada y traducida al castellano por Martín, Salvadó, Nadal, Miji, Rico, Lanz \& Taussig: Está conformada por 22 ítems que evalúan los efectos negativos sobre el cuidador en diferentes áreas de su vida (física, social, psíquica y económica). Cada ítem se evalúa mediante una escala tipo Likert con 5 modalidades de respuestas (de nunca hasta casi siempre). Tiene 3 subescalas: la primera evalúa el impacto subjetivo de la relación de cuidados sobre la vida del cuidador, la segunda evalúa los sentimientos de rechazo/hostilidad del cuidador hacia el paciente y la tercera evalúa la autovaloración de los cuidadores acerca de su competencia para mantener la relación de cuidado. Los rangos existentes son de 22-46: Sin sobrecarga; 47-55: Sobrecarga leve y de 56-110: Sobrecarga intensa. Además se aplicó el Cuestionario de Salud SF-36. 


\section{RESULTADOS}

De acuerdo a los análisis realizados se pueden señalar los siguientes resultados:

\section{Calidad de vida en pacientes}

En la tabla 4 se observa que en el instrumento específico Fact-G el puntaje en la escala global arroja una media de 60,0 con una desviación típica de 19,0. Este puntaje se clasifica en el rango de baja calidad de vida.

Respecto a las tres escalas globales del instrumento genérico SF-36 (escala de Estado Funcional, Escala de Bienestar y Escala de evaluación general de la salud) se indica que, tal como se observa en la tabla 5, el puntaje de la escala Estado Funcional arroja una media de 37,6 con una desvia- ción típica de 30,1 la cual se clasifica en el rango de baja calidad de vida, lo que sugiere que los problemas de salud física y emocional deterioran el funcionamiento físico cotidiano y las actividades sociales del paciente; el puntaje de la escala Bienestar arroja una media de 50,5 con una desviación típica de 23,3 la cual se clasifica en el rango de calidad de vida normal lo que refleja, en términos generales, que la población no percibe deterioro en relación a la vitalidad y a la sintomatología física (dolor) y mental (ansiedad y depresión) asociada a la enfermedad; por último, el puntaje de la escala Evaluación General de la Salud arroja una media de 62,3 con una desviación típica de 7,7 la cual se clasifica en el rango de alta calidad de vida, lo que indica que existe una adecuada percepción del estado de salud

\section{Tabla 4. Puntajes promedio de las escalas generales de calidad de vida, desde la perspectiva del paciente con cáncer en fase terminal}

\begin{tabular}{|l|c|c|c|c|c|}
\hline & N & Mínimo & Máximo & Media & Desv. típ. \\
\hline SF-36 Estado Funcional [Paciente] & 34 & 0,0 & 98,8 & 37,6 & 30,1 \\
\hline SF-36 Bienestar [Paciente] & 34 & 10,0 & 93,3 & 50,5 & 23,3 \\
\hline SF-36 Evaluación General de la Salud [Paciente] & 34 & 47,5 & 83,5 & 62,3 & 7,7 \\
\hline Puntuación Global FACT-G [Paciente] & 34 & 21 & 87 & 60,0 & 19,0 \\
\hline N válido (según lista) & 34 & & & & \\
\hline
\end{tabular}

Tabla 5. Puntajes promedio de las escalas generales de calidad de vida y sobrecarga emocional, desde la perspectiva del cuidador principal

\begin{tabular}{|l|c|c|c|c|c|}
\hline & N & Mínimo & Máximo & Media & Desv. típ. \\
\hline Puntaje Total Zarit' [Cuidador] & 34 & 26,0 & 72,0 & 46,0 & 12,2 \\
\hline SF-36 Estado Funcional [Cuidador] & 34 & 30,0 & 100,0 & 78,0 & 21,9 \\
\hline SF-36 Bienestar [Cuidador] & 34 & 17,3 & 95,6 & 63,1 & 19,0 \\
\hline SF-36 Evaluación General de la Salud [Cuidador] & 34 & 42,5 & 71,0 & 55,0 & 7,07 \\
\hline N válido (según lista) & 34 & & & & \\
\hline
\end{tabular}

1. Puntos de corte escala sobrecarga del cuidador Zarit: 22-46 Sin sobrecarga, 47-56 Sobrecarga leve, 57-110 Sobrecarga intensa. 
actual y la evolución de ésta a lo largo del tiempo.

\section{Calidad de vida en cuidadores}

Respecto a la calidad de vida de los cuidadores primarios, los resultados arrojan que de acuerdo al instrumento genérico SF-36 los puntajes para la escala global Estado Funcional y la escala Bienestar se ubican en el rango de alta calidad de vida, mientras que en la escala Evaluación General de la Salud el puntaje se ubica en el rango de calidad de vida normal (Tabla 5).

Por lo anterior se desprende que en términos generales los cuidadores poseen una buena percepción de la salud física y emocional, por lo que su funcionamiento físico cotidiano y las actividades sociales no se verían deteriorados. Asimismo, se percibe una adecuada vitalidad y ausencia de sintomatologías físicas.

Respecto al puntaje obtenido en el instrumento de sobrecarga del cuidador de Zarit, éste arroja una media de 46,0 con una desviación típica de 12,2 la cual se clasifica en el rango de "no hay sobrecarga", lo que revela, en términos generales, que los cuidadores no experimentan la vivencia de agotamiento en las áreas física, psíquica, social y económica de su vida (tabla 5). Sin embargo, al realizar una segmentación de los datos para evaluar la sobrecarga del cuidador según el estadio de funcionalidad (performance status) de los pacientes, se encontró que los puntajes de sobrecarga en los cuidadores de pacientes en estadio 4 de la Escala de funcionalidad Performance status de la enfermedad arrojaron una media de 54,2 con una desviación típica de 11,5 reflejando así una sobrecarga leve, lo cual permite concluir que los cuidadores de pacientes en estadios de funcionalidad más avanzados de la enfermedad presentan un leve grado de sobrecarga en las áreas física, social, psíquica y económica de su vida.

\section{Impacto de la relación de ayuda de cuidadores sobre la calidad de vida de pacientes}

Para determinar el impacto de la relación de ayuda de los cuidadores primarios sobre los pacientes que están a su cargo, se procedió a realizar análisis correlaciones entre las escalas globales del SF-36 y las subescalas del mismo instrumento (Función física, rol físico, dolor corporal, salud general, vitalidad, función social, rol emocional y salud mental), para ambas poblaciones. Una vez realizados los análisis de correlaciones se llevaron a cabo análisis de regresión múltiple para determinar el grado de influencia de aquellas escalas/subescalas que hayan obtenido correlaciones estadísticamente significativas en la primera fase de análisis. A partir de estos análisis se pudo evidenciar el impacto significativo que generaría la figura del cuidador en la calidad de vida de un paciente oncológico.

Los análisis señalan que en el caso de los pacientes, la escala general Estado Funcional correlacionaba en forma significativa $^{1}$ con las subescalas Dolor Corporal $(r$ $=0,401, p<0,05)$, Vitalidad $(r=0,379$, $p<0,05)$, Función Social $(r=0,499, p<$ $0,01)$, Salud Mental $(r=0,403, p<0,05)$, Bienestar $(r=0,356, p<0,05)$ y Evaluación General de la Salud ( $r=0,383, p<$ $0,05)$ del cuidador. Al realizar un análisis de regresión lineal múltiple la única que alcanzó un valor predictivo significativo fue Función Social del cuidador, explicando un $25 \%$ de la varianza $(\mathrm{F}=10,6 ; \mathrm{p}=$ $0,03)$ del Estado Funcional del paciente; esto quiere decir que la valoración que el cuidador tiene de su vida social en función de sus problemas de salud física y

1 Solo se mencionan las escalas y subescalas que hayan alcanzado una correlación igual o superior a 0,3 , puesto que según los parámetros estadísticos en ciencias sociales, dichos valores se consideran datos significativos. 
emocional, influye en la valoración que el paciente tiene acerca de su funcionamiento personal y social.

Asimismo, los puntajes obtenidos en la escala Estado Funcional del paciente fueron segmentados según el estadio de funcionalidad (performance status) de éstos, para luego realizar nuevamente un análisis de regresión lineal múltiple con las subescalas del cuidador: Dolor Corporal, Vitalidad, Función Social, Salud Mental, Bienestar y Evaluación General de la Salud y así determinar, a partir de esta segmentación, si se establece alguna relación predictiva entre las variables del cuidador antes mencionadas sobre el Estado Funcional del paciente. En concordancia con esto, se encuentra que la Vitalidad del cuidador explica un $82 \%$ de la varianza $(\mathrm{F}=$ 23,2; $p=0,005)$ del Estado Funcional de pacientes en estadio 3 de funcionalidad (performance status), esto quiere decir que la valoración que hacen los cuidadores de la energía y vitalidad que poseen, influye en la percepción que los pacientes de dicho estadio tienen acerca de su funcionamiento personal y social. Por otro lado, el Bienestar de los cuidadores explica un $62 \%$ de la varianza $(F=8,2 ; p=0,035)$ del Estado Funcional de los pacientes en estadio 4 de funcionalidad (performance status), esto quiere decir que la valoración que los cuidadores hacen acerca de su sintomatología física, mental y de su vitalidad, influye en la percepción que los pacientes de dicho estadio tienen sobre su funcionamiento personal y social.

De la misma forma, la escala general de Bienestar del paciente correlaciona positivamente con Función Social del cuidador $(r=0,342 p<0,05)$ Al ser sometida a una regresión lineal simple se señala que la Función Social explica el $12 \%$ de la varianza $(F=4,2 ; p=0,048)$ del Bienestar del paciente, es decir, la valoración que el cuidador hace de su vida social en función de su salud física y emocional, influye en la percepción que el paciente hace de su sintomatología física, mental y de su vitalidad.

Finalmente, la escala Evaluación General de la Salud del paciente correlacionó significativamente con Dolor Corporal $(r=$ 0,339, p < 0,05) y con Evaluación General de la Salud del cuidador $(r=0,403, p<$ $0,05)$; no obstante, al realizar un análisis de regresión lineal múltiple sólo la escala Evaluación General de la Salud del cuidador obtuvo un valor predictivo, explicando el $16 \%$ de la varianza $(F=6,2 ; p=0,018)$ de la escala Evaluación General de Salud del paciente, es decir, la valoración que el cuidador tiene acerca de su estado de salud actual y la evolución de ésta a lo largo del tiempo influye en la valoración que el paciente hace de su salud actual y de la evolución de ésta a lo largo del tiempo.

\section{DISCUSIÓN/CONCLUSIONES}

Es indudable que la evaluación de la calidad de vida en pacientes oncológicos presenta su mayor auge en la actualidad, puesto que se ha convertido en un problema de salud pública. En chile, dado el aumento de la prevalencia, las tasas de mortalidad y la reforma de salud surgida hace unos años, esta enfermedad ha sido incorporada al plan de garantías explicitas en salud, lo cual implica una cobertura especial de la misma. Los pacientes diagnosticados con cáncer terminal reciben una intervención integral y especializada, cuyo objetivo principal es contribuir a mejorar su calidad de vida. Es así como se incluyen prestaciones a los cuidadores primarios como un agente importante dentro del sistema a intervenir, puesto que éstos son los principales encargados de procurar el cuidado a los enfermos, lo que, a su vez, genera que sufran niveles de desgaste asociados a este cuidado ${ }^{(13,14)}$.

Investigaciones en torno al tema ${ }^{(13,25)}$ señalan que la calidad de vida tanto de 
pacientes oncológicos como de sus cuidadores se ve afectada producto de la situación de enfermedad, sin embargo, cabe destacar que dicha alteración no necesariamente repercute en las mismas áreas, ya que en los pacientes pareciera existir una tendencia al deterioro de las áreas física y funcional, mientras que en los cuidadores sería el área emocional ${ }^{(15,16)}$. Asimismo, en un informe reciente de la OMS sobre cuidados paliativos se afirma que hay evidencia de que la gente envejecida sufre innecesariamente debido a una carencia en la evaluación y tratamiento de sus problemas y por falta de acceso a cuidados paliativos ${ }^{(6)}$. Dichos hallazgos son concordantes con los resultados obtenidos en la presente investigación, ya que efectivamente la dimensión física y funcional del paciente se ven deterioradas significativamente, influyendo en la percepción que éstos tienen de su calidad de vida.

En el paciente la calidad de vida se ve influida de forma significativa por la Función Social, la Vitalidad y el Bienestar del cuidador; estos resultados sustentan que la valoración subjetiva que una de las poblaciones objetivo hace de su calidad de vida se ve estrechamente relacionada con la valoración subjetiva que la otra hace de la misma. Estos resultados son concordantes con los hallazgos encontrados en un estudio de Northouse, et al. ${ }^{(20)}$.

Por otra parte, la presencia de una sobrecarga leve en los cuidadores principales de pacientes en estadio 4 de la Escala de funcionalidad performance status tiene relación con el rol más activo que éstos adquieren en esta etapa de la enfermedad del paciente, en la cual éstos últimos pierden autonomía. Asimismo, esto guardaría relación con la cantidad de tiempo que los cuidadores llevarían ejerciendo su rol ${ }^{(12,17)}$.

Respecto a la valoración positiva que los cuidadores hacen de diversas áreas de su vida, ésta podría estar siendo explicada por el no reconocimiento de la sobrecarga que implica el cuidado de su familiar/paciente o el no reconocimiento de que su calidad de vida se ha visto deteriorada producto de esto mismo. La deseabilidad social es un factor que también podría influir en las respuestas dadas por los cuidadores, lo cual se relacionaría con la imagen positiva que éstos desearían proyectar respecto al rol de cuidado que ejercen.

Los pacientes hacen una valoración negativa de su calidad de vida global, lo cual estaría asociado a la situación de enfermedad por la que atraviesan, generando en éstos más problemas físicos, alteraciones en su vida cotidiana $y$, en definitiva, una percepción desfavorable de su salud ${ }^{(26) .}$

A partir de los resultados se constata que la figura de los cuidadores primarios genera un impacto significativo en la calidad de vida de los pacientes con cáncer avanzado. A partir de esto, se hace manifiesta la importancia de realizar investigaciones con ambas poblaciones $y$, más aún, considerar a los cuidadores en las líneas de intervención que se lleven a cabo desde el campo de la salud. Ello puesto que la presencia y bienestar de los mismos es un buen predictor de la calidad de vida de los pacientes a su cuidado. Los principales hallazgos del presente estudio indican que, en el caso de los pacientes, éstos perciben una baja calidad de vida a propósito de su estado funcional, mientras que los cuidadores perciben una alta calidad de vida en esta misma área y en su bienestar. No obstante, aquellos cuidadores que están a cargo de pacientes que se encuentran en estadio 4 de la Escala de funcionalidad performance status, es decir pacientes completamente discapacitados, postrados, que no pueden proveerse autocuidado y requieren cuidado de enfermería, presentan una sobrecarga leve. Esto puede explicarse justamente por el rol más activo que los cuidadores asumen en esta etapa de funcionalidad del paciente que tienen a cargo. 
Por otra parte, se observa que en el paciente oncológico, la calidad de vida se ve favorecida por el bienestar, función social y la vitalidad del cuidador. Esto quiere decir que la presencia de un cuidador que no percibe deterioro físico ni emocional, que posee una adecuada percepción de su salud, valoración positiva de su energía y vitalidad y posee una valoración positiva de su vida social contribuye a que el paciente perciba una mejor calidad de vida. La explicación radicaría en que el paciente percibiría más apoyo de su cuidador, al ser éste una persona con condiciones adecuadas para ejercer este rol. Son numerosos los testimonios que avalan la tesis de que la calidad de las relaciones interpersonales tienen un impacto enorme en la vida de los pacientes; siendo éstas un antecedente significativo para su salud ${ }^{(31)}$.

En relación a lo anterior, las líneas de acción en salud deberían orientarse a trabajar con cuidadores primarios fortaleciendo sus habilidades de afrontamiento, favoreciendo espacios de autocuidado y psicoeducando en cuanto a la entrega de información y estrategias de resolución de conflictos, para que éstos puedan ejercer un adecuado rol de cuidador ${ }^{(32)}$. Asimismo, los aspectos más estudiados en la literatura científica son los referentes al impacto de la enfermedad en las relaciones de pareja. Menos estudiadas han sido las relaciones entre padres e hijos y familia extensa ${ }^{(33)}$.

En el presente estudio se observaron algunas limitaciones las cuales se recomienda considerar para futuras investigaciones. Éstas guardan relación con el reducido tamaño de la muestra y la distribución de pacientes según su estadio en la Escala de funcionalidad Performance status, la cual fue de carácter heterogéneo. Por otro lado, se debe considerar que la calidad de vida es una evaluación de carácter temporal y está ligada al estado de salud actual de la persona ${ }^{(11,12)}$. Por lo tanto, estos resultados podrían variar ya sea en forma positi- va como negativa, de allí la importancia de mantener evaluaciones constantes de dicho constructo sobre todo en pacientes oncológicos en fase terminal, dadas las características de la rápida evolución de la enfermedad en esta fase, así como también el vertiginoso paso de un estadio de funcionalidad a otro.

\section{Agradecimientos}

Los autores agradecen a la Unidad del Dolor y Cuidados Paliativos del Hospital en Red Dr. Juan Noé Crevani de la ciudad de Arica, Chile, al equipo médico, técnicos y funcionarios de dicho dispositivo de salud que contribuyeron a este proyecto, por su interés y apoyo permanente.

Hacemos extensivo un especial reconocimiento a todos los pacientes y sus familias que tan generosamente aceptaron ser partícipes de este estudio. Finalmente, agradecemos al convenio de Desempeño UTA-MINEDUC.

\section{REFERENCIAS BIBLIOGRÁFICAS}

1. Organización Mundial de la Salud. Centro de Prensa, 2012. Nota descriptiva № 297, cáncer. En línea. [Acceso 31 marzo 2012]. Disponible en: http://www.webcitation. org/query? url=http $\% 3 \mathrm{~A} \% 2 \mathrm{~F} \% 2 \mathrm{Fwww}$. who.int $\% 2$ Fmediacentre $\% 2$ Ffactsheets $\% 2$ Ffs297\%2Fes\%2F\&date=2012-08-06

2. Organización Mundial de la Salud. Datos estadísticos, 2007. Cifras destacables: el impacto del Cáncer. En línea. [Acceso 15 de junio 2007]. Disponible en: http://www.webcitation.org/ query? url=http $\% 3 \mathrm{~A} \% 2 \mathrm{~F} \% 2 \mathrm{Fwww}$.who.int $\% 2$ Fresearch $\% 2$ Fes $\% 2 F \&$ date $=2012-08-06$

3. MINSAL, Unidad de Cáncer. Programa de alivio del dolor y cuidados paliativos. Informe anual, Octubre, 2005.

4. López-lbor B. Aspectos médicos, psicológicos y sociales del cáncer Infantil. Psicooncología 2009; 6(2):281-4. 
5. Prieto M, Gil E, Heierle C y Frías A. La perspectiva de las cuidadoras informales sobre la atención domiciliaria. Un estudio cualitativo con ayuda de un programa informático. Rev Esp Salud Pública 2002;76:613-25.

6. Bórquez P, Romero C. El paciente oncológico geriátrico. Rev Chil Cir 2007; 59(6):467-71. Doi: 10.4067/S071840262007000600015

7. Eastern Cooperative Oncology Group. ECOG Performance Status, 2006. En línea. [Acceso 15 marzo 2008]. Disponible en: http://www.webcitation.org/ query? url=http $\% 3 \mathrm{~A} \% 2 \mathrm{~F} \% 2 \mathrm{Fecog}$. dfci. harvard.edu\%2 Fgeneral\%2 Fperf_stat. html\&date=2012-08-06.

8. Astudillo A, Mendinueta A. Necesidades de los cuidadores del paciente crónico. Guía de recursos sanitarios y sociales en la fase final de la vida en Gipuzkoa. Séptima sección Madrid, 2008. p. 235-55.

9. Orley J, Kuyken W. Quality of life assessment: international perspectives. Berlin: Springer-Verlag, 1994.

10. Serra-Sutton V. Desarrollo de la versión española del Child Health and Illness Profile para medir el estado de salud percibido en la adolescencia. Tesis doctoral. Facultad de Medicina de la Universidad Autónoma de Barcelona. Barcelona-España. Consultado septiembre 13, 2011. Disponible en: http://tdx.cat/bitstream/handle/10803/4621/vss1de2.pdf?sequence=1

11. Arraras JI, Martínez M, Manterota A., Laínez N. La evaluación de la calidad de vida del paciente oncológico. El grupo de calidad de vida de la EORTC. Psicooncología 2004; 1(1):87-98.

12. Padierna C, Fernández C, González A. Calidad de vida en pacientes oncológicos terminales, medida con el QL-CA-AFEX. Psicothema 2002; 14(1):1-8.

13. Matthews A, Baker F, Spillers R. Family caregivers'quality of life: Influence of health protective stance and emotional strain. Psychol Health 2004; 19(5): 62541. Doi: 10.1080/0887044042000205594
14. Uribe ZP. Manual de apoyo para personas que brindan apoyo a otras personas. México: Secretaría de Salud, 2006.

15. Barrón BS, Alvarado S. Desgaste físico y emocional del cuidador primario en cáncer. Cancerología 2009; 4:39-46.

16. Torres F, Beltrán G, Martínez P, Saldivar G, Quesada C, Cruz T. Cuidar a un enfermo ¿pesa? Rev de Divulgación Científica de la Universidad Veracruzana. 2006; 19

17. Krikorian A, Vélez M, González O, Palacio C, Vargas J. La experiencia de sufrimiento en cuidadores de pacientes con dolor oncológico y no oncológico de tres instituciones de la ciudad de Medellín, Colombia. Av Enferm 2010; (28):91-7.

18. Romero E. La familia del paciente paliativo. "Hablan las cuidadoras", 2008. En línea. [Acesso 20 mayo 2010]. Disponible en: http://www.webcitation. org/query? url=http $\% 3 \mathrm{~A} \% 2 \mathrm{~F} \% 2 \mathrm{Fhuma}$ nizar.es\%2Fuploads\%2Fmedia\%2F08_ atencion_a_la_familia_del_enfermo_XIII_ Jorn.pdf\&date=2012-08-06

19. Expósito Y. La calidad de vida en los cuidadores primarios de pacientes con Cáncer. [revista en la Internet]. 2008 Sep [Acceso 2013 marzo 29]; 7(3). Disponible en: http://scielo.sld.cu/scielo. php?script=sci_arttext \&pid=S1 729 519X2008000300004\&lng=es

20. González M, Pizarro M. Efecto de tres modalidades de intervención terapéutica sobre la calidad de vida subjetiva de pacientes oncológicos. Tesis de pregrado para optar al Título de Psicólogo, Departamento de Filosofía y Psicología, Universidad de Tarapacá, Arica, Chile. 1997.

21. Northouse L, Mood D, Templin T, et al. Couples' patterns of adjustment to colon cancer. Soc Sci Med 2000; 50:271-84.

22. Pérez C. Equipo multidisciplinario de atención a pacientes oncológicos en la atención primaria de salud. Rev Cubana Oncol 2000; 16(3):58-62.

23. Schwartzmann L. Calidad de vida relacionada con la salud: aspectos conceptuales. 
Revista Ciencia y Enfermería 2003; 9(2):921.

24. Northouse L, Mood D, Kershaw T, Schafenacker A, Mellon S, Walker J, et al. Quality of life of woman recurrent breast cancer and their family members. J Clin Oncol 2002; 20:4050-60. Doi: 10.1200/ JCO.2002.02.054.

25. Ringdal G, Ringdal K, Jordhey M, Kaasa S. Does social support from family and friends works as a buffer against reactions to stressfull life events such as terminal cancer? Palliat Support Care 2007; 5:61-9.

26. González A, Fernández C, García G, Soler J, Arce C, Cueto J. Parámetros de calidad de vida en pacientes oncológicos terminales en hospitalización domiciliaria. Psicothema 2001; 13(2):310-7.

27. Frost $M$, Suman $V$, Rummans $T$, Dose AM, Taylor M, Novotny P, et al. Physical, psychological and social well-being of women with breast cancer: The influence of disease phase. Psychooncology 2000; 9:221-31. Doi:10.1002/1099$1611(200005 / 06) 9: 3<221::$ AID PON456>3.0.CO;2-T

28. Gutiérrez T, Raich R, Sánchez D, Deus J. Instrumentos de evaluación en psicología de la salud. Madrid: Alianza Editorial, 2003.
29. Unidad de Investigación Recursos Sanitarios del IMIM. Ficha técnica del instrumento SF-36v2. En línea. [Acceso 7 de junio 2007] Disponible en http://www.webcitation.org/query? url=http $\% 3 \mathrm{~A} \% 2 \mathrm{~F} \% 2$ Firyss. imim.es $\% 2$ Firys s $\% 2$ Fnew-ficha. asp\%3Fid\%3D37+\&date=2012-08-06

30. Martín M, Salvadó I, Nadal S, Miji LC, Rico JM, Lanz P, Taussig MI. Adaptación para nuestro medio de la Escala de Sobrecarga del Cuidador (Caregiver Burden Interview) de Zarit. Rev Gerontologist 1996;6:338-46.

31. Navas C, Villegas H, Hurtado R, Zapata E. Programa de intervención psicoeducativo para el apoyo del paciente con cáncer. Rev Venez Oncol 2007; 19(1):84-93.

32. De la Huerta R, Corona J, Méndez V. Evaluación de los Estilos de Afrontamiento en Cuidadores primarios de niños con cáncer. Neurol Neurocir Psiquiat 2006;39(1):4651.

33. Grau C, Espada M. Percepciones de los padres de niños enfermos de cáncer sobre los cambios en las relaciones familiares. Psicooncología 2012;9(1):125-36. Doi:10.5209/rev_PSIC.2012.v9.n1.39142 